\title{
Favorable Effects of 24-Week Whole-Body Vibration on Glycemic Control and Comprehensive Diabetes Therapy in Elderly Patients with Type 2 Diabetes
}

\author{
Takumi Kitamoto (D) - Ryuichi Saegusa · Takuma Tashiro • \\ Tomomi Sakurai $\cdot$ Koutaro Yokote $\cdot$ Takahiko Tokuyama
}

Received: March 2, 2021 / Accepted: April 22, 2021 / Published online: May 12, 2021

(C) The Author(s) 2021

\section{ABSTRACT}

Background: Elderly patients with type 2 diabetes (T2DM) are vulnerable to treatment-inducible hypoglycemia, falls, and depressive symptoms. Although it is challenging for elderly patients to adhere to regular exercise, its positive effect on functional ability, glycemic control, and mental wellness offers comprehensive diabetes treatment. In the present study, we aimed to investigate a novel exercise approach for the elderly, focusing on wholebody vibration (WBV).

Methods: This retrospective cohort study was conducted in a primary-care setting at a medical fitness center affiliated with the incorporated

Supplementary Information The online version contains supplementary material available at https:// doi.org/10.1007/s13300-021-01068-0.

T. Kitamoto $(\square) \cdot$ T. Sakurai · T. Tokuyama

Tokuyama Clinic, Chiba, Japan

e-mail: tk2752@cumc.columbia.edu

T. Kitamoto · R. Saegusa · T. Tashiro · T. Sakurai ·

T. Tokuyama

Medicalfitness TOKU, Chiba, Japan

T. Kitamoto $\cdot$ K. Yokote

Department of Endocrinology, Hematology and Gerontology, Chiba University Graduate School of Medicine, Chiba, Japan

T. Kitamoto

Division of Endocrinology, Department of

Medicine, Columbia University, New York, USA medical institution of THY (TOTAL HEALTH YARD). Fourteen (WBV group) and 12 (control group) elderly patients with T2DM undergoing and not undergoing our WBV program, respectively, for $>6$ months were analyzed. Primary endpoints were the functional ability changes, evaluated by Timed Up and Go (TUG), Sit-toStand test (SST), gait length, and grip test. Secondary endpoints were global glycemic control and questionnaires, namely the Diabetes Treatment Satisfaction Questionnaire (DTSQ) and Geriatric Depression Scale (GDS). All records of exercise adherence and any adverse events were followed.

Results: Significant improvements in TUG and SST were found only in the WBV group [TUG: $7.1 \pm 0.9,7.1 \pm 0.8$ to $7.0 \pm 1.0,6.6 \pm 0.9$ (s), $P=0.63,0.01 ;$ SST: $10.4 \pm 1.9,11.3 \pm 2.4$ to $9.7 \pm 2.3, \quad 9.5 \pm 2.1 \quad$ (s) $, \quad P=0.62, P<0.01$, control vs. WBV group, respectively]. The WBV group demonstrated significant improvement of hemoglobin A1C levels $(7.2 \pm 0.8$ to $6.9 \pm 0.5, P<0.01)$ and DTSQ and GDS scores, while the control group did not. There were no hypoglycemic events during the study. The WBV program adherence was $93.3 \pm 8.0 \%$.

Conclusion: We demonstrated the favorable effect of WBV training on balance, diabetes treatment, and mood. Therefore, WBV training can be proposed as comprehensive therapy in a safe manner and potentially has a positive effect on health-related quality of life in elderly patients with T2DM. 
Keywords: Elderly patients; Type 2 diabetes; Whole-body vibration; Exercise; Fall; Hypoglycemia; Balance

\section{Key Summary Points}

Why carry out this study?

Comprehensive treatment for elderly patients with type 2 diabetes is warranted to benefit their health-related quality of life (QOL)

Although regular exercise has the potential to improve both physical and mental health, it is challenging for elderly patients because of their broad backgrounds

Does a whole-body vibration (WBV)-based exercise program work as a well-tolerated, safe, and comprehensive type 2 diabetes treatment for elderly patients?

What was learned from the study?

A 3-month intervention of our WBV program on top of exercise counseling can improve the functional ability of balance in elderly patients with type 2 diabetes

A 6-month intervention of our WBV program ameliorated global glycemic control without changing fast glucose levels and hypoglycemic adverse events

Our WBV program increased diabetes treatment-related satisfaction and decreased the depression score

The adherence to our WBV program was $>$ $90 \%$

The WBV exercise program provides comprehensive diabetes therapy for elderly patients with type 2 diabetes to benefit health-related QOL

\section{DIGITAL FEATURES}

This article is published with digital features, including a summary slide to facilitate understanding of the article. To view digital features for this article go to https://doi.org/10.6084/ m9.figshare.14465391.

\section{INTRODUCTION}

The prevalence of diabetes increases with age. With the rapid growth of the elderly population, $>25 \%$ of Japanese patients with type 2 diabetes (T2DM) are $>65$ years of age. The healthy life expectancy of elderly patients with T2DM is less than that of people without diabetes because of the increased rate of falls $[1,2]$ and cognitive decline [3]. Diabetes treatment-related hypoglycemia and depressive symptomatology are closely associated with these features [4-6]. An improvement in psychiatric conditions and physical function can reduce the risk of falls. Besides better glycemic control, providing regular exercise can reduce the risk of falls by improving both the physical and mental health of these patients [7-10]. In this context, welltolerated regular exercise for elderly patients with T2DM is warranted to benefit their healthrelated quality of life.

Although studies are limited, stretching and balance training have been found to improve joint flexibility and reduce the risk of falls in elderly patients with T2DM $[11,12]$. Additionally, a recent large cohort study demonstrated that balance exercises, based on Tai Chi, are more effective than conventional exercises in preventing falls in elderly adults [13]. However, many elderly patients are unwilling or unable to complete individually targeted exercise [14]. Given the increased comorbidities in elderly patients with T2DM compared with those in the healthy population, adherence to regular exercise is a greater challenge for them. Therefore, developing a novel exercise program that is both practical and easy to adhere to is necessary.

Whole-body vibration (WBV) training has been shown to have potential in improving balance in non-diabetic elderly individuals 
[15-17]. WBV is only effective when the individual's entire weight is placed on a plate that creates mechanical vibrations. This stimulates the muscle spindles and sensory nerves. The burden on the cardiopulmonary system is less than that from conventional resistance training [18]. Therefore, WBV enhances the efficacy of exercise, leading to improved physical function of the individual with a light load. In the present study, we investigated a new exercise approach focusing on WBV in a comprehensive diabetes treatment strategy in elderly patients with T2DM. We evaluated the effects of a 6-month WBV training program in diabetic treatment, balance, and depressive symptoms in elderly patients with T2DM in a primary-care setting. Furthermore, we determined patients' adherence to this training program.

\section{METHODS}

\section{Patients}

This study has a retrospective cohort design. Study participants were examined at the incorporated THY (TOTAL HEALTH YARD) medical institution between April 2016 and May 2017. The protocol was approved by the joint ethical review board (Table S1. Approval number: 14000050. 20180615-4629), and written informed consent was obtained from all participants for this study. This study was performed in accordance with the Helsinki Declaration of 1964 and its later amendments. THY manages both the Tokuyama Clinic and medical fitness center TOKU. Five certified diabetes educators and certified health fitness programmers at each institution cooperatively provide exercise therapy, comprising a counseling and WBV training program. We provide exercise counseling to patients with diabetes by certified health fitness programmers in our clinic. They instructed fitness programs that were feasible in each physical condition and social background. In the present study, the patients with T2DM who began participating in the WBV exercise program on top of exercise counseling at our medical fitness center during the study term (WBV group) were reviewed. The details of our
WBV training program (Power-Plate pro6 + TM, USA) are summarized in Table S2. The patients who received exercise counseling in the same manner as the WBV group and did not receive any other intervention at our institutions were evaluated as the control group. All participants were older than 65 years, diagnosed with T2DM according to the guidelines of the Japan Diabetes Society, and lived independently. None of the patients changed their diabetes medication and their target glycemic control in this study period. No subjects had severe complications, such as autonomic neuropathy, proliferative diabetic retinopathy, endstage nephropathy, and major cardiovascular diseases. We scrutinized the first 15 patients in each group from the beginning of the study period in a sequential manner. As three cases in the control group and one case in the WBV group moved out of our cohort during the study period, 12 and 14 subjects who had undergone exercise therapy for $>6$ months were analyzed.

\section{Sample Size}

We referred to two prior randomized control studies for sample size calculation, which demonstrated improvement of TUG after 6 and 12 weeks of the whole-body vibration intervention $[19,20]$. Based on these studies, we anticipated a 0.8 -s improvement in the TUG test with a standard deviation of 0.8 by whole-body vibration if the intervention was appropriately provided for $>3$ months. We calculated that at least 28 participants (14 per group) would provide $70 \%$ power to detect a difference of $0.8 \mathrm{~s}$ as significant at a two-sided 5\% significance level.

\section{Outcome Measures}

The primary outcome measure of the study was functional ability. This composite endpoint was assessed through the Timed Up and Go (TUG) test, sit-to-stand test (SST), and step length. The secondary outcome measures were the HbA1c level, fast plasma glucose level, C-peptide immunoreactivity (CPR) index, and the scores obtained through the questionnaires of the Diabetes Treatment Satisfaction Questionnaire 
(DTSQ) and the Geriatric Depression Scale 15 (GDS). The changes in patients' functional ability from baseline to 6 months after the intervention were evaluated. In addition to TUG, SST, and step length, we also evaluated handgrip. For the TUG test, participants were asked to stand up from a chair, walk $3 \mathrm{~m}$, cross a marked line on the floor, turn around to walk back, and sit down on the chair [21]. The SST was performed by requiring them to stand up and sit down five times, as quickly as possible, with arms folded across their chests [22]. The average time was recorded. We used an average of the two trials to determine step length and handgrip. All blood and urine tests were performed by Kotobiken Medical Laboratories Inc. The HbA1c level was expressed as the international standard value. We calculated the C-peptide immunoreactivity (CPR) index as follows: fasting CPR $(\mathrm{ng} / \mathrm{ml}) /$ fasting plasma glucose $(\mathrm{mg} / \mathrm{dl}) \times 100$ [23]. Patients' treatment satisfaction levels were determined using a Japanese version of DTSQ [24]. DTSQ scores were calculated based on eight items, of which six evaluated the patients' satisfaction, while the rest evaluated hyperglycemia and hypoglycemia. Mood status was assessed using the Japanese version of the GDS, with a score of 0 being the best and 15 being the worst [25]. Adverse events were defined as any undesirable experiences reported by the medical staff and patients during the study. Adherence to the WBV training was calculated as the number of completed sessions divided by the total number of sessions. Sessions were provided thrice a week.

\section{Data Analysis}

Variables with normal and abnormal distributions were expressed as the mean \pm SD and the median [interquartile range (IQR)]. The Student $t$-test or Mann-Whitney $U$ test was used for comparisons between the groups. A one-way repeated measure ANOVA was performed to compare each group's mean differences in three time points with Bonferroni correction. The relative proportions of categorical variables were assessed using the Yates $\chi^{2}$ test or Fisher exact test. A $P$ value $<0.05$ was considered statistically significant. All data analyses were performed using JMP 11 (SAS Institute Inc., Cary, NC, USA).

\section{RESULTS}

Data collected from 12 and 14 patients were analyzed following a 6-month intervention in the control and WBV groups, respectively. The assessment of functional capacity demonstrated a significant improvement in the Timed Up and Go test (TUG) $[7.1 \pm 0.9,7.1 \pm 0.8$ to $7.0 \pm 1.0$, $6.6 \pm 0.9$ (s), $P=0.63,0.01$, control vs. WBV group, respectively] and sit-to-stand tests (SST) $[10.4 \pm 1.9,11.3 \pm 2.4$ to $9.7 \pm 2.3,9.5 \pm 2.1$ (s), $P=0.62, P<0.01]$ in the WBV group. However, no improvement was observed in the control group. Gait length was significantly improved $[62.8 \pm 7.2,64.9 \pm 7.2$ to $70.6 \pm 8.1$, $71.5 \pm 7.5(\mathrm{~cm}), P<0.005,<0.005]$ and handgrip was unchanged in both groups (Fig. 1). All the clinical characteristics at baseline did not show a significant difference between the groups (Table 1). Body mass index was significantly lower in the control group $[23.1 \pm 3.7$ to $22.8 \pm 3.6 ; \quad P=0.019]$, while systolic blood pressure $[131.4 \pm 13.7$ to $124.9 \pm 11.9 \mathrm{mmHg}$; $P=0.038]$, aspartate transaminase $[25.7 \pm 10.0$ to $23.5 \pm 7.9 ; P=0.044]$, alanine aminotransferase $[28.1 \pm 19.0$ to $22.4 \pm 12.5 ; P=0.038]$, and HbA1c [7.2 \pm 0.8 to $6.9 \pm 0.5 \% ; P=0.004]$ were significantly decreased only in the WBV group after the intervention (Table 1). Fast plasma glucose levels and CPR indices were unchanged in both groups. The patients' treatment satisfaction levels were significantly increased, and concerns about hyperglycemia were decreased in the WBV group. A significant improvement in depressive symptoms was observed in the WBV group (Table 2). To see the detailed transition of the significant changes of primary and secondary outcomes seen in the WBV group, we added the 3-month data of the functional capacity, $\mathrm{HbA1c}$, fast plasma glucose level, CPR index, DTSQ, and GDS scores in this analysis. We found that the changes in the functional capacity of TUG and SST occurred as early as 3 months after WBV intervention, while the changes in gait length in 6 months (Figure S1). In contrast to TUG and SST, HbA1c and 

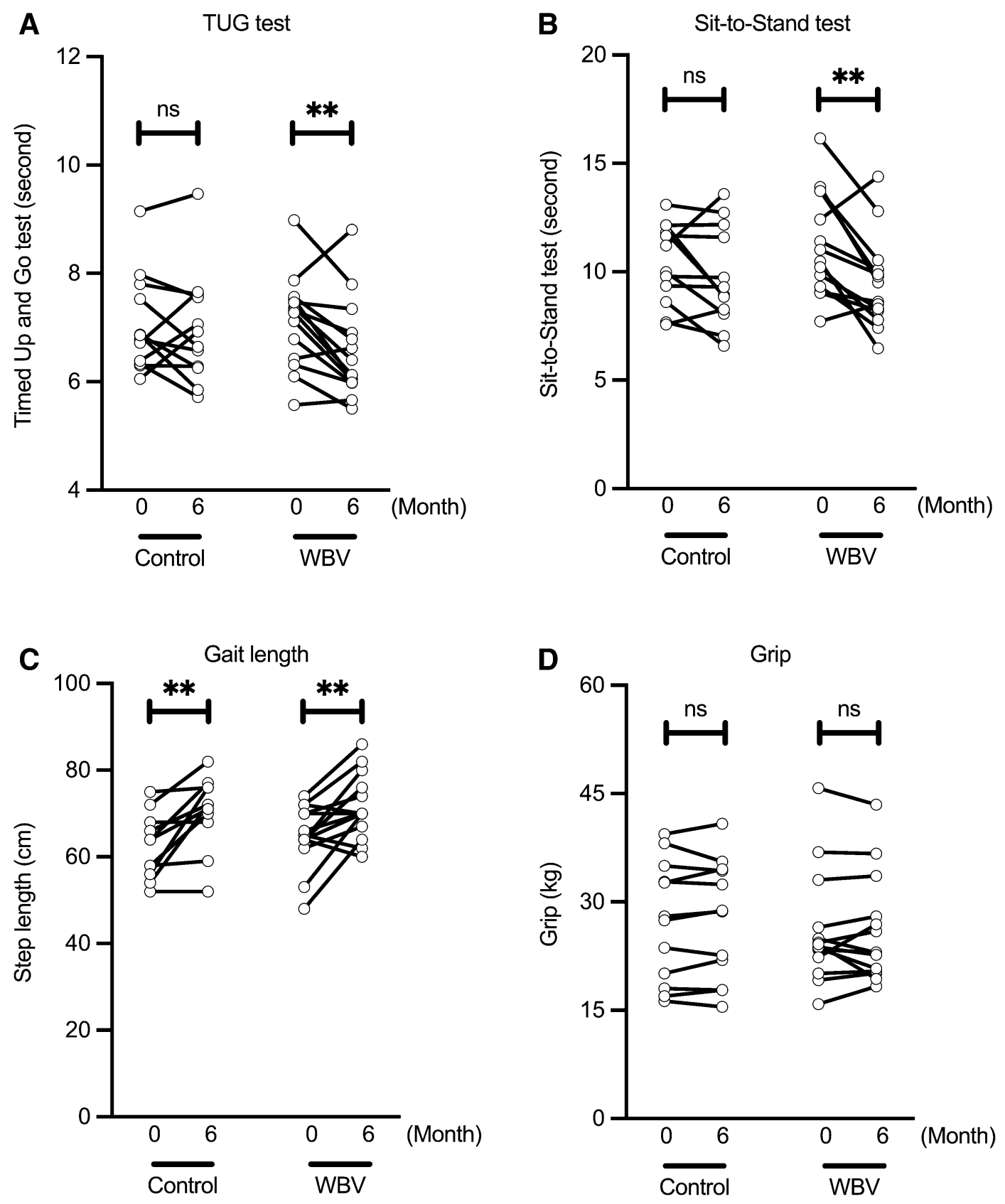

Fig. 1 Changes in the functional capacity before and after the intervention. Each point shows the individual score obtained in each test in the control and WBV groups. A significant improvement was observed in the TUG and sitto-stand tests in the WBV group, while the gait length

improved significantly in both groups. Handgrip was unchanged in both groups. The $P$ values demonstrate the statistical difference before and after the intervention in each group. ${ }^{*} P<0.05,{ }^{* *} P<0.01$. ns not significant

the DTSQ and GDS scores required 6 months to improve (Table S3 and S4). No adverse events, such as hypoglycemia, occurred in any of the

cases. Adherence was $93.3 \pm 8.0 \%$ in the WBV group (Table S5). 
Table 1 Comparison of the basic clinical characteristics between the control and WBV groups

\begin{tabular}{|c|c|c|c|c|c|c|c|}
\hline & \multicolumn{2}{|c|}{ Control group $(n=12)$} & \multirow[b]{2}{*}{$\begin{array}{l}P \text {-value } \\
\text { (within } \\
\text { group) }\end{array}$} & \multicolumn{2}{|c|}{ WBV group $(n=14)$} & \multirow[b]{2}{*}{$\begin{array}{l}P \text {-value } \\
\text { (within } \\
\text { group) }\end{array}$} & \multirow[b]{2}{*}{$\begin{array}{l}P \text {-value } \\
\text { (between } \\
\text { groups) }\end{array}$} \\
\hline & Pre & Post & & Pre & Post & & \\
\hline Sex (male/female) & $5 / 7$ & & & $10 / 4$ & & & \\
\hline Age (years) & $68.8 \pm 3.8$ & & & $69.6 \pm 3.3$ & & & \\
\hline $\begin{array}{l}\text { Duration of } \\
\text { T2DM (years) }\end{array}$ & $9.5(1.8-17.0)$ & & & $11.5(5.5-13.5)$ & & & \\
\hline $\begin{array}{l}\text { Number of } \\
\text { medications for } \\
\text { T2DM }\end{array}$ & $2(0.25-2.75)$ & & & $1.5(0.75-2.25)$ & & & \\
\hline Body mass index & $23.1 \pm 3.7$ & $22.8 \pm 3.6$ & 0.019 & $24.8 \pm 5$ & $24.8 \pm 5$ & 0.914 & 0.035 \\
\hline $\mathrm{SBP}(\mathrm{mmHg})$ & $136.3 \pm 9.2$ & $133.7 \pm 12.9$ & 0.427 & $131.4 \pm 13.7$ & $124.9 \pm 11.9$ & 0.038 & 0.366 \\
\hline $\mathrm{DBP}(\mathrm{mmHg})$ & $79.6 \pm 8.8$ & $75.9 \pm 7.7$ & 0.125 & $74.9 \pm 7.1$ & $72.9 \pm 12.2$ & 0.445 & 0.631 \\
\hline PR (beats/min) & $74.7 \pm 11.6$ & $77.3 \pm 11.3$ & 0.274 & $71.1 \pm 10.3$ & $71 \pm 8.2$ & 0.795 & 0.206 \\
\hline AST & $22.3 \pm 6.1$ & $26.9 \pm 16.8$ & 0.228 & $25.7 \pm 10$ & $23.5 \pm 7.9$ & 0.044 & 0.056 \\
\hline ALT & $22.2 \pm 9.8$ & $34 \pm 37.7$ & 0.209 & $28.1 \pm 19$ & $22.4 \pm 12.5$ & 0.040 & 0.016 \\
\hline G-GTP & $30.8 \pm 16.4$ & $43.9 \pm 34.3$ & 0.107 & $46.5 \pm 41.5$ & $32.8 \pm 21.6$ & 0.158 & 0.026 \\
\hline Uric acid & $5.2 \pm 1$ & $5.6 \pm 1.1$ & 0.086 & $4.9 \pm 1.1$ & $4.9 \pm 1.1$ & 0.740 & 0.181 \\
\hline $\begin{array}{l}\text { Serum creatinine } \\
(\mathrm{mg} / \mathrm{dl})\end{array}$ & $0.8 \pm 0.4$ & $0.9 \pm 0.4$ & 0.202 & $0.7 \pm 0.1$ & $0.7 \pm 0.2$ & 0.072 & 0.353 \\
\hline $\begin{array}{l}\mathrm{eGFR}(\mathrm{ml} / \mathrm{min} / \\
\left.1.73 \mathrm{~m}^{2}\right)\end{array}$ & $71.2 \pm 17.1$ & $68.7 \pm 17.2$ & 0.445 & $74.7 \pm 10.6$ & $73.6 \pm 11.6$ & 0.554 & 0.574 \\
\hline $\begin{array}{l}\text { Urine albumin to } \\
\text { creatinine ratio } \\
(\mathrm{mg} / \mathrm{g})\end{array}$ & $\begin{array}{l}10.5 \\
\quad(7.1-108)\end{array}$ & $\begin{array}{l}10.7 \\
\quad(8.2-126.1)\end{array}$ & 0.139 & $\begin{array}{l}18.9 \\
\quad(4.7-52.9)\end{array}$ & $\begin{array}{l}14.3 \\
\quad(7.6-39.2)\end{array}$ & 0.229 & 0.903 \\
\hline TG (mg/dl) & $100.4 \pm 42.4$ & $107.5 \pm 61.8$ & 0.692 & $90.2 \pm 27.9$ & $94.2 \pm 40.7$ & 0.551 & 0.855 \\
\hline HDL-C (mg/dl) & $61.7 \pm 19.7$ & $62.1 \pm 16.5$ & 0.906 & $62.8 \pm 22$ & $62.6 \pm 21.1$ & 0.902 & 0.861 \\
\hline LDL-C (mg/dl) & $97.8 \pm 23.8$ & $105.2 \pm 33.9$ & 0.225 & $119.3 \pm 29.6$ & $117.1 \pm 24.8$ & 0.639 & 0.208 \\
\hline HbAlc (\%) & $6.8 \pm 0.5$ & $6.9 \pm 0.6$ & 0.452 & $7.2 \pm 0.8$ & $6.9 \pm 0.5$ & 0.004 & 0.876 \\
\hline $\begin{array}{l}\text { Fasting plasma } \\
\text { glucose }(\mathrm{mg} / \mathrm{dl})\end{array}$ & $135 \pm 17$ & $134.9 \pm 28.1$ & 0.566 & $143 \pm 36$ & $138.2 \pm 27.6$ & 0.274 & 0.450 \\
\hline CPR index & $1.4 \pm 0.4$ & $1.3 \pm 0.3$ & 0.219 & $1.7 \pm 0.8$ & $1.6 \pm 0.5$ & 0.107 & 0.608 \\
\hline
\end{tabular}


Table 2 Changes in diabetes treatment-related QOL and depression scale

\begin{tabular}{|c|c|c|c|c|c|c|}
\hline & \multicolumn{2}{|c|}{ Control group $(n=12)$} & \multirow[b]{2}{*}{$P$-value } & \multicolumn{2}{|c|}{ WBV group $(n=14)$} & \multirow[b]{2}{*}{$P$-value } \\
\hline & Before & After & & Before & After & \\
\hline \multicolumn{7}{|l|}{ DTSQ } \\
\hline Total score (nos. $1,4-8$ ) & $29(24-31)$ & $28.5(25-35)$ & 0.391 & $27(23-29)$ & $31(28-33)$ & 0.028 \\
\hline No. 2 frequency of hyperglycemia & $3(1-4)$ & $2(0-3)$ & 0.125 & $3(1-5)$ & $2(0-2)$ & 0.004 \\
\hline No. 3 frequency of hypoglycemia & $0(0-1)$ & $0(0-0)$ & 1.000 & $0(0-0)$ & $0(0-1)$ & 0.500 \\
\hline Depression (GDS) & $1.5(0.3-3.8)$ & $2.0(1.0-2.0)$ & 0.828 & $1.5(1.0-4.5)$ & $1(0-1)$ & 0.016 \\
\hline
\end{tabular}

The changes in the DTSQ and GDS scores from baseline to 24 weeks after the intervention are shown as sub-clusters, such as total treatment satisfaction (nos. 1, 4-8) and frequency of hyperglycemia and hypoglycemia. Data are expressed as median (interquartile range)

$D T S Q$ diabetes treatment satisfaction questionnaire, GDS geriatric depression scale

\section{DISCUSSION}

The present study clearly demonstrated that our 6-month WBV program effectively improved the physical and mental health of elderly patients with T2DM at a primary-care setting, and it was well tolerated in these patients. The three primary benefits of the WBV program were improved glycemic control, balance, and an improvement in depressive symptoms. To the best of our knowledge, this is the first trial that has demonstrated the beneficial effects of WBV, which may confer comprehensive beneficial treatment effects in elderly patients with T2DM.

The strength produced during standing and walking might play a crucial role in improving balance. WBV enhances the strength and motor control of the quadriceps in a short period [26]; however, the current evidence regarding the use of WBV training to improve balance and mobility in older adults remains unclear [27-29]. In the present study, patients who received the original WBV program (Table S2) acquired better balance and mobility indicated by their improvement in the TUG and STT in 3 months. This effect continues for 6 months (Figure S1) [21]. By contrast, gait length was similarly improved in 6 months, and handgrip did not change in either group. The faster time indicated better functional performance in TUG and SST, and all of these tests are indicators of fall risk. Longer than $13.5 \mathrm{~s}$ in the TUG test predicts future falling risk [30], and longer than $13.6 \mathrm{~s}$ in SST predicts increased disability and morbidity [31]. For gait length, every $10 \mathrm{~cm}$ length decrease increases the fall risk by $9.5 \%$ [32], and reduced gait length was also suggested to be associated with fear [33]. In the present study, almost all of the cases showed less than these cut-off values in TUG and SST, except two and four patients in the control and WBV group in SST. In this situation, we assumed that significant improvement from baseline is clinically meaningful change. These results suggested that $>3$ months of WBV intervention contributed to improving balance and mobility, reducing the risk of falls. However, we need to confirm these results by following up on the cases to determine the future frequency of falls. The changes in gait length seemed to be accomplished by the weekly exercise counseling itself. These results suggested that both groups were well motivated to start exercise in their daily life based on the feasible exercise instructions given through their counseling. As gait length is also affected by fear, confidence in their day-to-day management would result in longer gait length at the test. On the other hand, the bodyweight reduction seen in the control group might not be a good sign, as there is no other evidence of the improvement of physical function than gait length. We need to give careful nutritional therapy instructions to these patients. 
In the present study, the WBV group had a significant decrease in the glycemic level. However, the participants in the current cohort demonstrated well-controlled glucose levels at baseline for their ages. Exercise-induced hypoglycemia is one of the major concerns, especially in elderly patients, which increases the risk of falling and cognitive dysfunction $[3,34,35]$. In the present study, the WBV group patients showed a significant improvement in $\mathrm{HbA1c}$, although their fasting glucose levels and CPR indexes were unchanged. Also, there were no hypoglycemic episodes. The improvement in global glycemia without reducing fasting glycemia suggested that their glucose levels were optimized in a smaller range in the fasting and postprandial glucose transition after the intervention. WBV may affect glycemic control via several mechanisms. In a future study, evaluation of glucose and insulin levels in response to nutrients and basal glucose and insulin levels would help confirm these findings.

Improvement of motor function and amelioration of liver function might contribute toward glycemic control. Better patient quality of life is known to be related to better glycemic control [36]. Considering the significant improvement in both DTSQ and GDS scores after the WBV intervention (Table 2), the current WBV program may enhance optimal glycemic control by positively affecting motivation and adherence to diabetic treatment. The unchanged lipid metabolism may be because of the high frequency of statin use before enrollment in both groups. Of those enrolled in the study, eight $(66.7 \%)$ patients in the control group and eight $(57.1 \%)$ patients in the WBV group had been prescribed a statin, which enabled favorable lipid control. The reason why any statistical difference in LDL levels between groups could not be seen regardless of more patients being prescribed statins in the control group was partly because we prescribed statins based on the same target LDL level of $<120 \mathrm{mg}$ / $\mathrm{dl}$ in the participants in this study in addition to the small sample size.

In the present study, depressive symptoms were reduced exclusively in the WBV group. The potential of exercise in treating depression has been shown by other forms of exercise in prior studies $[9,10]$. Additionally, our data shed light on the novel insight of using WBV for depression, which can assist in preventing falls in these patients.

Our WBV program was performed thrice a week, with 15-min personalized-intensity sessions. This regimen had $>90 \%$ adherence during the 6-month intervention. Therefore, it is appropriate for elderly patients.

This study has several limitations, such as a retrospective cohort design, small sample size, and single-center setting. Although both groups' baseline characteristics seemed similar, we should carefully consider the confounding bias, such as patients' favoring WBV and the sex imbalance of male dominance in the WBV group. To address these limitations, future multicenter randomized controlled trials consisting of a larger sample size are warranted. We need to set hard endpoints, such as falls, in a more extended period to confirm that the findings in the present study are clinically significant and reproducible changes. We hope this study can facilitate larger scale research involving more clinics and contribute to healthrelated quality of life in elderly patients with diabetes through the novel exercise program.

\section{CONCLUSION}

In conclusion, the application of a 6-month WBV exercise program in a primary-care setting was well tolerated, safe, and potentially effective at glycemic control, preventing falls through improved balance and mood in elderly patients with T2DM. These findings can promote clinical studies to evaluate the effect of WBV in elderly patients with T2DM and will encourage the use of this novel exercise-based management.

\section{ACKNOWLEDGEMENTS}

We thank all the participants of the study. We acknowledge Naoko Miyake for her management of the staff and her valuable advice for the WBV program. 
Funding. There was no role of a sponsor. All the cost of the present study, including the study and the Rapid Service Fee, was owed by Tokuyama Clinic.

Medical Writing. This manuscript has been edited by Vikas Narang from Editage to ensure language and grammar accuracy and is errorfree. The cost was paid by Tokuyama Clinic.

Disclosures. All the authors, Takumi Kitamoto, Ryuichi Saegusa, Takuma Tashiro, Tomomi Sakurai, Koutaro Yokote, and Takahiko Tokuyama, have nothing to disclose.

Authors' Contribution. Takumi Kitamoto designed the study, analyzed the results, and wrote the manuscript. Takuma Tashiro and Ryuichi Saegusa generated a whole-body vibration program, instructed the patients, and provided counseling; Tomomi Sakurai acquired the patients' informed consents and collected and analyzed data. Koutaro Yokote edited the manuscript. Takahiko Tokuyama supervised the study and edited the manuscript.

Compliance with Ethics Guidelines. The protocol in the present study was approved by the joint ethical review board (IRB ID number provided by Japan Ministry of Health Labour and Welfare, 1,400,050: approval number: 14000050. 20,180,615-4629: The name of Ethics Review Committee, Masao Morita). This study was performed in accordance with the Helsinki Declaration of 1964 and its later amendments and that consent to participate and publish was obtained from all patients.

Data Availability. The datasets generated during and/or analyzed during the current study are available from the corresponding author on reasonable request.

Open Access. This article is licensed under a Creative Commons Attribution-NonCommercial 4.0 International License, which permits any non-commercial use, sharing, adaptation, distribution and reproduction in any medium or format, as long as you give appropriate credit to the original author(s) and the source, provide a link to the Creative Commons licence, and indicate if changes were made. The images or other third party material in this article are included in the article's Creative Commons licence, unless indicated otherwise in a credit line to the material. If material is not included in the article's Creative Commons licence and your intended use is not permitted by statutory regulation or exceeds the permitted use, you will need to obtain permission directly from the copyright holder. To view a copy of this licence, visit http://creativecommons.org/licenses/by$\mathrm{nc} / 4.0 /$.

\section{REFERENCES}

1. Yau RK, Strotmeyer ES, Resnick HE, et al. Diabetes and risk of hospitalized fall injury among older adults. Diabetes Care. 2013;36(12):3985-91.

2. Napoli N, Strotmeyer ES, Ensrud KE, et al. Fracture risk in diabetic elderly men: the MrOS study. Diabetologia. 2014;57(10):2057-65.

3. Crane PK, Walker R, Larson EB. Glucose levels and risk of dementia. N Engl J Med. 2013;369(19): 1863-4.

4. Trief PM. Depression in elderly diabetes patients. Diabetes Spectrum. 2007;20(2):71-5.

5. Park M, Reynolds CF 3rd. Depression among older adults with diabetes mellitus. Clin Geriatr Med. 2015;31(1):117-37.

6. Kvelde T, Lord SR, Close JCT, et al. Depressive symptoms increase fall risk in older people, independent of antidepressant use, and reduced executive and physical functioning. Arch Gerontol Geriatr. 2015;60(1):190-5.

7. Gillespie LD, Robertson MC, Gillespie WJ, et al. Interventions for preventing falls in older people living in the community. Cochrane Database Syst Rev. 2012;9:2.

8. Li F, Eckstrom E, Harmer P, Fitzgerald K, Voit J, Cameron KA. Exercise and fall prevention: Narrowing the research-to-practice gap and enhancing integration of clinical and community practice. J Am Geriatr Soc. 2016;64(2):425-31.

9. Schuch FB, Vancampfort D, Richards J, Rosenbaum $\mathrm{S}$, Ward PB, Stubbs B. Exercise as a treatment for 
depression: a meta-analysis adjusting for publication bias. J Psychiatr Res. 2016;77:42-51.

10. Kirk-Sanchez NJ, McGough EL. Physical exercise and cognitive performance in the elderly: current perspectives. Clin Interv Aging. 2014;9:51-62.

11. Herriott MT, Colberg SR, Parson HK, Nunnold T, Vinik AI. Effects of 8 weeks of flexibility and resistance training in older adults with type 2 diabetes. Diabetes Care. 2004;27(12):2988-9.

12. Morrison S, Colberg SR, Mariano M, Parson HK, Vinik AI. Balance training reduces falls risk in older individuals with type 2 diabetes. Diabetes Care. 2010;33(4):748-50.

13. Li F, Harmer P, Fitzgerald K, et al. Effectiveness of a therapeutic Tai Ji Quan intervention vs a multimodal exercise intervention to prevent falls among older adults at high risk of falling: a randomized clinical trial Tai Ji Quan vs a multimodal exercise intervention for preventing falls in high-risk older adults Tai Ji Quan vs a multimodal exercise intervention for preventing falls in high-risk older adults. JAMA Intern Med. 2018;178(10):1301-10.

14. Nyman SR, Victor CR. Older people's participation in and engagement with falls prevention interventions in community settings: an augment to the Cochrane systematic review. Age Ageing. 2012;41(1):16-23.

15. Bogaerts ACG, Delecluse C, Claessens AL, Troosters $\mathrm{T}$, Boonen S, Verschueren SMP. Effects of whole body vibration training on cardiorespiratory fitness and muscle strength in older individuals (a 1-year randomised controlled trial). Age Ageing. 2009;38(4):448-54.

16. Lai C-C, Tu Y-K, Wang T-G, Huang Y-T, Chien K-L. Effects of resistance training, endurance training and whole-body vibration on lean body mass, muscle strength and physical performance in older people: a systematic review and network metaanalysis. Age Ageing. 2018;47(3):367-73.

17. Lam FMH, Liao LR, Kwok TCY, Pang MYC. Effects of adding whole-body vibration to routine day activity program on physical functioning in elderly with mild or moderate dementia: a randomized controlled trial. Int J Geriatr Psychiatry. 2018;33(1): 21-30.

18. Torvinen S, Kannus P, Sievanen $H$, et al. Effect of four-month vertical whole body vibration on performance and balance. Med Sci Sports Exerc. 2002;34(9):1523-8.

19. del Pozo-Cruz B, Alfonso-Rosa RM, del Pozo-Cruz J, Sanudo B, Rogers ME. Effects of a 12-wk whole-body vibration based intervention to improve type 2 diabetes. Maturitas. 2014;77(1):52-8.

20. Kordi Yoosefinejad A, Shadmehr A, Olyaei G, Talebian S, Bagheri H, Mohajeri-Tehrani MR. Shortterm effects of the whole-body vibration on the balance and muscle strength of type 2 diabetic patients with peripheral neuropathy: a quasi-randomized-controlled trial study. J Diabetes Metab Disord. 2015;14:45.

21. Podsiadlo D, Richardson S. The timed "Up \& Go": a test of basic functional mobility for frail elderly persons. J Am Geriatr Soc. 1991;39(2):142-8.

22. Guralnik JM, Simonsick EM, Ferrucci L, et al. A short physical performance battery assessing lower extremity function: association with self-reported disability and prediction of mortality and nursing home admission. J Gerontol. 1994;49(2):M85-94.

23. Iwata M, Matsushita Y, Fukuda K, et al. Secretory units of islets in transplantation index is a useful predictor of insulin requirement in Japanese type 2 diabetic patients. J Diabetes Investig. 2014;5(5): 570-80.

24. Ishii H. The Japanese version of the Diabetes Treatment Satisfaction Questionnaire (DTSQ): translation and clinical evaluation. J Clin Exp Med. 2000;192:809-14.

25. Sugishita K, Sugishita M, Hemmi I, Asada T, Tanigawa T. A validity and reliability study of the Japanese version of the geriatric depression scale 15 (GDS-15-J). Clin Gerontol. 2017;40(4):233-40.

26. Filippi GM, Brunetti O, Botti FM, et al. Improvement of stance control and muscle performance induced by focal muscle vibration in young-elderly women: a randomized controlled trial. Arch Phys Med Rehabil. 2009;90(12):2019-25.

27. Sitja-Rabert M, Rigau D, Fort Vanmeerghaeghe A, Romero-Rodriguez D, Bonastre Subirana M, Bonfill $\mathrm{X}$. Efficacy of whole body vibration exercise in older people: a systematic review. Disabil Rehabil. 2012;34(11):883-93.

28. Orr R. The effect of whole body vibration exposure on balance and functional mobility in older adults: a systematic review and meta-analysis. Maturitas. 2015;80(4):342-58.

29. Lam FM, Chan PF, Liao L, et al. Effects of wholebody vibration on balance and mobility in institutionalized older adults: a randomized controlled trial. Clin Rehabil. 2018;32(4):462-72.

30. Shumway-Cook A, Brauer S, Woollacott M. Predicting the probability for falls in community- 
dwelling older adults using the timed up \& go test. Phys Ther. 2000;80(9):896-903.

31. Guralnik JM, Ferrucci L, Pieper CF, et al. Lower extremity function and subsequent disability: consistency across studies, predictive models, and value of gait speed alone compared with the short physical performance battery. J Gerontol A Biol Sci Med Sci. 2000;55(4):M221-231.

32. Verghese J, Holtzer R, Lipton RB, Wang C. Quantitative gait markers and incident fall risk in older adults. J Gerontol A Biol Sci Med Sci. 2009;64(8): 896-901.

33. Maki BE. Gait changes in older adults: predictors of falls or indicators of fear? J Am Geriatr Soc. 1997;45(3):313-20.
34. Schwartz AV, Vittinghoff E, Sellmeyer DE, et al. Diabetes-related complications, glycemic control, and falls in older adults. Diabetes Care. 2008;31(3): 391-6.

35. Johnston SS, Conner C, Aagren M, Ruiz K, Bouchard J. Association between hypoglycaemic events and fall-related fractures in Medicare-covered patients with type 2 diabetes. Diabetes Obes Metab. 2012;14(7):634-43.

36. Ishii $\mathrm{H}$, Anderson JH Jr, Yamamura A, Takeuchi $\mathrm{M}$, Ikeda I. Improvement of glycemic control and quality-of-life by insulin lispro therapy: Assessing benefits by ITR-QOL questionnaires. Diabetes Res Clin Pract. 2008;81(2):169-78. 